\title{
Walker's Cancellation Theorem
}

\author{
Robert Lubarsky and Fred Richman \\ Florida Atlantic University
}

\begin{abstract}
Walker's cancellation theorem says that if $B \oplus \mathbf{Z}$ is isomorphic to $C \oplus \mathbf{Z}$ in the category of abelian groups, then $B$ is isomorphic to $C$. We construct an example in a diagram category of abelian groups where the theorem fails. As a consequence, the original theorem does not have a constructive proof. In fact, in our example $B$ and $C$ are subgroups of $\mathbf{Z}^{2}$. Both of these results contrast with a group whose endomorphism ring has stable range one, which allows a constructive proof of cancellation and also a proof in any diagram category.
\end{abstract}

\section{Cancellation}

An object $G$ in an additive category is cancellable if whenever $B \oplus G$ is isomorphic to $C \oplus G$, then $B$ is isomorphic to $C$. Walker [6] and independently Cohn [3] answered a question of Kaplansky by showing that finitely generated abelian groups are cancellable in the category of abelian groups. The most interesting case is that of the integers $\mathbf{Z}$. That's because finitely generated groups are direct sums of copies of $\mathbf{Z}$ and of cyclic groups of prime power order, and a cyclic group of prime power order has a local endomorphism ring, hence is cancellable by a theorem of Azumaya [2].

It is somewhat anomalous that $\mathbf{Z}$ is cancellable. A rank-one torsion-free group $A$ is cancellable if and only if $A \cong \mathbf{Z}$ or the endomorphism ring of $A$ has stable range one [1, Theorem 8.12], [4. (A ring $R$ has stable range one if whenever $a R+b R=R$, then $a+b R$ contains a unit of $R$.) Thus for rank-one torsion-free groups, the endomorphism ring tells the whole story - except for $\mathbf{Z}$. It turns out that an object is cancellable if its endomorphism ring has stable range one. The proof of this in [5, Theorem 4.4] is constructive and works for any abelian category. It is also true, [5], that semilocal rings have stable range one, so Azumaya's theorem is a special case of this. In fact, that the endomorphism ring of $A$ has stable range one is equivalent to $A$ being substitutable, a stronger condition than cancellation [5, Theorem 4.4]. We say that $A$ is substitutable if any two summands of a group, with complements that are isomorphic to $A$, have a common complement. The group $\mathbf{Z}$ is not substitutable: Consider the subgroups of $\mathbf{Z}^{2}$ generated by $(1,0),(0,1),(7,3)$, and $(5,2)$. The first and second, and the third and fourth, are complementary summands. The second and fourth do not have a common complement because that would require $(a, b)$ with $a= \pm 1$ and $2 a-5 b= \pm 1$.

In this paper we will investigate whether $\mathbf{Z}$ is cancellable in the (abelian) category $\mathcal{D}_{T}(\mathbf{A b})$ of diagrams of abelian groups based on a fixed finite poset $T$ with a least element. There is a natural embedding of $\mathbf{A b}$ into $\mathcal{D}_{T}(\mathbf{A} \mathbf{b})$ given by taking a group into the constant diagram on $T$ with identity maps between the groups on the nodes. In particular, we can identify the group of integers as an object of $\mathcal{D}_{T}(\mathbf{A b})$. As the endomorphism ring of any group $G$ is the same as that of its avatar in $\mathcal{D}_{T}(\mathbf{A b})$, a substitutable group is substitutable viewed as an object in $\mathcal{D}_{T}(\mathbf{A b})$. However it turns out that $\mathbf{Z}$ is not cancellable in $\mathcal{D}_{T}(\mathbf{A b})$ where $T$ is the linearly ordered set $\{0,1,2\}$.

It follows that Walker's theorem does not admit a constructive proof, because the construction can be viewed as a Kripke model counter-example. In fact, it is not even provable for $B$ and $C$ subgroups of $\mathbf{Z}^{2}$. It was the question of whether Walker's theorem had a constructive proof that initiated our investigation. (Most any proof of Azumaya's theorem is constructive.) 
As any homomorphism from an abelian group onto $\mathbf{Z}$ splits, Walker's theorem can be phrased as follows: If $A$ is an abelian group, and $f, g: A \rightarrow \mathbf{Z}$ are epimorphisms, then ker $f \cong \operatorname{ker} g$. The following theorem gets us part way to a proof of Walker's theorem.

Theorem 1. Let $A$ be an abelian group and $f, g: A \rightarrow \mathbf{Z}$ be epimorphisms. Then $f(\operatorname{ker} g)=$ $g(\operatorname{ker} f)$ so that

$$
\frac{\operatorname{ker} g}{\operatorname{ker} f \cap \operatorname{ker} g} \cong f(\operatorname{ker} g)=g(\operatorname{ker} f) \cong \frac{\operatorname{ker} f}{\operatorname{ker} f \cap \operatorname{ker} g}
$$

Thus we get the desired isomorphism $\operatorname{ker} f \cong \operatorname{ker} g$ if $\operatorname{ker} f \cap \operatorname{ker} g=0$ or if $f(\operatorname{ker} g)$ is projective. Classically, every subgroup of $\mathbf{Z}$ is projective, so this constitutes a classical proof.

\section{The example}

Our example lives in the category $\mathcal{D}_{T}(\mathbf{A b})$ of diagrams of abelian groups based on the linearly ordered set $T=\{0,1,2\}$. The example shows that you can't cancel $\mathbf{Z}$ in $\mathcal{D}_{T}(\mathbf{A b})$.

The groups on the nodes will be subgroups $A_{0} \subset A_{1} \subset A_{2}=\mathbf{Z}^{3}$ defined by generators:

$$
A_{0}=\begin{gathered}
(1,3,0) \\
(3,1,0)
\end{gathered} \quad A_{1}=\begin{gathered}
(1,0,-24) \\
(0,1,8) \\
(0,0,64)
\end{gathered} \quad A_{2}=\begin{aligned}
& (1,0,0) \\
& (0,1,0) \\
& (0,0,1)
\end{aligned}
$$

Note that $(0,8,0),(8,0,0) \in A_{0}$. The maps between these groups are inclusions. Define the maps $f, g: \mathbf{Z}^{3} \rightarrow \mathbf{Z}$ by $f(a, b, c)=a$ and $g(a, b, c)=b$. The maps $f$ and $g$ each induce maps from these three groups into $\mathbf{Z}$ which give two maps from the diagram into the constant diagram $\mathbf{Z}$. We denote the kernel of the map $f$ restricted to $A_{i}$ by $\operatorname{ker}_{i} f$ and similarly for $g$. These kernels admit the following generators:

$$
\begin{gathered}
\operatorname{ker}_{0} f=(0,8,0) \quad \operatorname{ker}_{1} f=\begin{array}{c}
(0,1,8) \\
(0,0,64)
\end{array} \quad \operatorname{ker}_{2} f=\begin{array}{l}
(0,1,0) \\
(0,0,1)
\end{array} \\
\operatorname{ker}_{0} g=(8,0,0) \quad \operatorname{ker}_{1} g=\begin{array}{c}
(1,0,-24) \\
(0,0,64)
\end{array} \quad \operatorname{ker}_{2} g=\begin{array}{l}
(1,0,0) \\
(0,0,1)
\end{array}
\end{gathered}
$$

The diagrams $B=\operatorname{ker} f$ and $C=\operatorname{ker} g$ are clearly each embeddable in the diagram $\mathbf{Z} \oplus \mathbf{Z}$. That $B \oplus \mathbf{Z}$ is isomorphic to $C \oplus \mathbf{Z}$ follows from the fact that the diagram $A$ can be written as an internal direct sum $B \oplus \mathbf{Z}$ and also as an internal direct sum $C \oplus \mathbf{Z}$. The generator of $\mathbf{Z}$ in the first case is the element $(1,3,0)$, in the second case $(3,1,0)$.

Theorem 2. There is no isomorphism between $\operatorname{ker} f$ and $\operatorname{ker} g$ in $\mathcal{D}_{T}(\mathbf{A b})$.

The following result shows that we can't get an example that is a subobject of the diagram $\mathbf{Z}^{n}$ using the linearly ordered set $T=\{0,1\}$.

Theorem 3. Let $T=\{0,1\}$. In the category $\mathcal{D}_{T}(\mathbf{A b})$, if $A$ and $B$ are subobjects of $\mathbf{Z}^{n}$, and $A \oplus \mathbf{Z}$ is isomorphic to $B \oplus \mathbf{Z}$, then $A$ is isomorphic to $B$.

This theorem leaves open the question of whether there is an counterexample of this sort using the poset that looks like a "V". 


\section{Canceling $\mathrm{Z}$ with respect to subgroups of $\mathrm{Q}$}

So we can't cancel $\mathbf{Z}$ with respect to certain subgroups of $\mathbf{Z} \oplus \mathbf{Z}$. What is the situation is with respect to subgroups of $\mathbf{Z}$ ? Constructively:

Theorem 4. Let $B$ be an abelian group such that every nontrivial homomorphism from $B$ to $\mathbf{Z}$ is one-to-one. If $f$ is a homomorphism from $B \oplus \mathbf{Z}$ onto $\mathbf{Z}$, then $\operatorname{ker} f$ is isomorphic to $m B$ for some positive integer $m$. Hence if $B$ is torsion free, then $\operatorname{ker} f$ is isomorphic to $B$.

Note that any torsion-free group $B$ of rank at most one satisfies the hypothesis of the theorem.

What other groups $B$ allow cancellation of $\mathbf{Z}$ ? It suffices that $B$ be finitely generated. To see this, look at Theorem 1 . If ker $f$ is finitely generated, then $g(\operatorname{ker} f)$ is a finitely generated subgroup of $\mathbf{Z}$, hence is projective. From this argument it suffices that any image of $B$ in $\mathbf{Z}$ be finitely generated. Notice that subgroups of $\mathbf{Z}$ need not have this property. What about a direct sum of two groups that allow cancellation of $\mathbf{Z}$, such as a direct sum of two subgroups of $\mathbf{Z}$ ?

\section{References}

[1] ARnold, DAvid M., Finite rank torsion free abelian groups and rings, Lecture Notes in Mathematics 931, Springer, 1982.

[2] Azumaya, Goro, Corrections and supplementaries to my paper concerning Krull-Remak-Schmidt's thm, Nagoya Math. J, 1 (1950), 117-124.

[3] Cohn, Paul M., The complement of a finitely generated direct summand of an abelian group, Proc. Amer. Math. Soc., 7 (1956), 520-521.

[4] Fuchs, Laszlo and Frans Loonstra, On the cancellation of modules in direct sums over Dedekind domains, Indag. Math. 33 (1971), 163-169.

[5] Lam, Tsit Yuen, A crash course on stable range, cancellation, substitution, and exchange, math.berkeley.edu/ lam/ohio.ps

[6] Walker, Elbert A., Cancellation in direct sums of groups, Proc. Amer. Math. Soc., 7 (1956) 898-902. 